\title{
Changes in lipid composition associated with electronic cigarette use
}

\author{
Holly R. Middlekauff ${ }^{1,2 \dagger}$, Kevin J. William ${ }^{1,3}$, Baolong Su ${ }^{1,3}$, Kacey Haptonstall' ${ }^{1}$, Jesus A. Araujo 1,2,4 , Xiaohui Wu ${ }^{1,4}$,
} Jason $\mathrm{Kim}^{1,4}$ and Tamer Sallam ${ }^{1,2,4^{*}+}$ (D)

\begin{abstract}
Background: Electronic cigarette use is on the rise despite a number of reports linking electronic cigarettes with adverse health outcomes. Recent studies have suggested that alterations in lipid signaling may be one mechanism by which electronic cigarettes contribute to lung pulmonary function. Vitamin E acetate, for example, is synthetic form of Vitamin E transported via lipids, found to be associated with electronic cigarette associated lung injury. Lipids are absolutely critical for normal lung physiology and perturbations in a number of lipid pathways have been associated with respiratory illness. Is it conceivable that electronic cigarette use even in seemingly healthy cohorts are associated with alterations in lipid pathways?

Methods: To investigate quantitative alterations in the plasma lipidome associated with electronic cigarette use in healthy we obtained plasma samples from 119 male and female participants with who were either: (1) chronic tobacco cigarette (TC) smokers (> 12 months of self-reported TC use), (2) chronic Electronic cigarette (EC) users (>12 months of self-reported EC use), or (3) non-users. We measured quantitative lipid species across different lipid sub-classes from plasma samples using the Sciex Lipidyzer.
\end{abstract}

Results: We found that male and female tobacco and electronic cigarette users had distinct lipidome signatures across a number of lipid species although the vast majority of lipids were unchanged when compared to non-users. Intriguingly, we found that female but not male electronic cigarette users had lower levels of plasmalogens, critical glycerophospholipids secreted by alveoli and required for normal surfactant function.

Conclusions: In summary, our study does not reveal striking changes associated with electronic cigarette use but we observed sex-specific changes in lipids known to be critical for lung function.

Keywords: Lipids, Tobacco, Electronic cigarettes

\section{Background}

Electronic cigarettes (ECIGS) were originally introduced in 2004 as a safe alternative to tobacco cigarettes (TCIGS). In less than a decade from their introduction, ECIG use has increased by $5000 \%$ and recent estimates project that ECIG use will surpass TCIG use in two

\footnotetext{
*Correspondence: tsallam@mednet.ucla.edu

${ }^{\dagger}$ Holly R. Middlekauff and Tamer Sallam contributed equally to this work

1 Division of Cardiology, Department of Medicine, University of California, Los Angeles, CA, USA

Full list of author information is available at the end of the article
}

decades [1-3]. More recently, a rapidly growing number of reports have linked ECIG use with a number of adverse health events including acute or subacute lung disease [4-6]. The pattern of injury appears to be distinct from adverse effects of TCIGs on lung disease lending weight to the notion that liquid constituents of ECIGS such as propylene glycol, glycerin, flavorant(s) or other components beyond nicotine may be involved in mechanisms of lung injury.

Significant heterogeneity is observed in the pattern and severity of lung injury associated with ECIG use which hints that diverse mechanisms may be at play. Although 
the precise mechanism(s) of ECIG induced lung injury remain poorly understood, there are two prevailing theories. First, that components of ECIGS cause a direct chemical injury to the lungs [7]. A second hypothesis is that ECIG use is associated with alterations in normal lung physiology that predispose to lung disease [8]. In a recent preclinical study in female mice, ENDS exposure led to alterations in lung phospholipid composition and innate immune responses independent of nicotine [9]. Intriguingly, a subset of vaping associated pulmonary diseases have been associated with lipid overload of immune cells and more recent evidence suggested that changes in specific lipids such as the isoprenoid Vitamin $E$ acetate is associated with acute lung injury $[8,10]$. The later phenomenon is often referred to as E-cigarette or Vaping Product Use-Associated Lung Injury (EVALI) and is thought to be uncommon in vapers who do not vape THC. Interestingly, liquid components of ECIGS such as propylene glycol and glycerol can theoretically interfere with critical lipid metabolic reactions but whether ECIG use in humans is associated with perturbations in lipid homeostasis and how ECIG use compare with TCIG use remains unexplored.

Lipids are critical for normal lung physiology since they play a direct role in membrane structure integrity, cell-signaling, oxidative stress responses and surfactant synthesis [11, 12]. For example, perturbations in a glycerophospholipid known as plasmalogen secreted by pneumocytes has been associated with pulmonary diseases [13]. In this study of otherwise healthy, young males and females, we aimed to investigate quantitative alterations in plasma lipidome associated with chronic ECIG use compared to chronic TCIG use and nonsmoking. We validate some of the previously observed effects of TCIG use on lipid composition and find that ECIG use is associated with unique sexually dimorphic signatures in lipid species that is independent from nicotine use. Notably, we observe that ECIG use in females is associated with lower level of lipids critical for surfactant function.

\section{Methods}

Plasma samples were obtained during the period of 2015-2018 in a cohort of subjects participating in our clinical investigations (NCT03072628, NCT02740595, NCT02724241) of tobacco product use.

\section{Human Subjects}

In these studies [14], plasma samples were obtained from healthy male and female subjects, ages $21-45$ years, who were: 1 ) chronic ( $\geq 12$ months) TCIG smokers, 2) chronic ( $\geq 12$ months) ECIG users (no dual users), or 3) non-smokers. End-tidal CO was measured in ECIG users to detect those who were surreptitiously using TCIGs, and if $\mathrm{CO}>10 \mathrm{ppm}$ was detected, the subjectwas eliminated from the study. Exclusion Criteria: Nonsinus rhythm (e.g. atrial fibrillation); competitive or trained athletes; pregnant women; taking prescription medications regularly (oral contraceptives were allowed); more than 2 alcoholic drinks per day; using illicit drugs, including marijuana, or a positive urine toxicology test; chronic illness, including asthma, hypertension, heart disease, diabetes; hyperlipidemia; obesity $(\mathrm{BMI}>30)$; or regular exposure to secondhand smoke in non-TCIG smokers. The experimental protocol was approved by the Institutional Review Board at the University of California, Los Angeles, and written informed consent was obtained from each participant.

\section{Experimental protocol}

Participants were asked to abstain from food, caffeine, exercise and tobacco product use for at least $12 \mathrm{~h}$ prior to the study. Participants were placed in a supine position in a quiet, temperature-controlled $\left(21^{\circ} \mathrm{C}\right)$ room in the Human Physiology Laboratory located in the University of California, Los Angeles Clinical Translational Research Center. Blood was drawn in pre-iced heparinized vacutainers and placed on ice. Blood was centrifuged to separate into plasma samples, which were frozen at $-80{ }^{\circ} \mathrm{C}$.

\section{Lipid analysis}

$25 \mu \mathrm{l}$ of plasma was pipetted into a glass tube for extraction. A modified Bligh and Dyer extraction (Bligh, 1959) is carried out on samples. Prior to biphasic extraction, a 13-lipid subclass Lipidyzer Internal Standard Mix is added to each sample $(A B$ Sciex, 5040156). Following two successive extractions, pooled organic layers are dried down in a Genevac EZ-2 Elite. Lipid samples are resuspended in 1:1 methanol/ dichloromethane with $10 \mathrm{mM}$ Ammonium Acetate and transferred to robovials (Thermo 10800107) for analysis. Samples are analyzed on the Sciex Lipidyzer Platform for targeted quantitative measurement of 1100 lipid species across 13 subclasses. Differential Mobility Device on Lipidyzer was tuned with SelexION tuning kit (Sciex 5040141). Instrument settings, tuning settings, and MRM list available upon request. Data analysis performed on Lipidyzer software. Quantitative values were normalized to volume. Samples were analyzed on the Sciex Lipidyzer Platform for targeted quantitative measurement of lipids. 


\section{Statistics}

We used ANOVA with correction for statistical analysis of lipid composition between the three groups. Principal component analysis was done using ClusterVis [15]. The input data is the lipid concentration from our lipidomic run measurements and the PCA calculation is based on pcaMethods [15].

\section{Results}

Our study enrolled 77 male and 42 female participants. Baseline demographic information of participants is included in Table 1. Targeted serum lipidomics measurement across 1000 different lipid species was performed (Fig. 1a). Principal component analysis (PCA), a technique that summarizes the variation in samples and strong patterns in datasets, showed significant overlap between samples across groups for both males (Fig. 1b) and females (Fig. 1c) consistent with the notion that TCIG or ECIG use do not globally alter lipid composition. For both male and female subjects TCIG users contributed to more variance than ECIG or nonsmokers with largest difference in variance across the first component (explains $48 \%$ of all variation) between TCIG and non-smokers (Fig. 1).

Sample distribution of bonds and carbon group for lipid is shown in Figs. 2, 3. Analysis of major classes of lipids across species showed sex-specific trends. In line with previous studies, female and male non-users showed differences in levels of diacylglycerol (DAG), triacylglycerol (TAG) and Lysophosphatidylcholine (LPC). Consistent with previous studies male TCIG smokers showed an increase in total cholesterol esters (CE) species compared with nonuser and ECIG user groups (Fig. 4) [16]. Male participants also showed an increase in ceramides (CER) and hexosylceramides (HCER) in TCIG groups compared with others (Fig. 4). These results are consistent with previous reports that tobacco increases ceramide biosynthesis due to activation of inflammatory signaling $[17,18]$. Female participants showed an increase in diacylglyceroles (DAG) and triacylglycerols (TAG) in the ECIG group (Fig. 5). Similar trends were seen for the TCIG group for DAG and TAG although it did not reach statistical significance. Taken together, our results suggest that the vast majority of lipid species are unchanged comparing TCIG or ECIG users with non-smokers however there are striking sexually dimorphic differences driven by TCIG or ECIG group.
Table 1 Demographic data on study participants

\begin{tabular}{|c|c|c|c|}
\hline & Control & EC-user & TC-user \\
\hline \multicolumn{4}{|l|}{$\mathrm{N}$} \\
\hline Male & 26 & 27 & 24 \\
\hline Female & 19 & 15 & 8 \\
\hline \multicolumn{4}{|l|}{ Age mean (STD) } \\
\hline Male & $26.4(4.3)$ & $27.1(5.1)$ & $27.1(6.4)$ \\
\hline Female & $28.9(7.0)$ & $30(5.5)$ & $27.5(4.33)$ \\
\hline \multicolumn{4}{|l|}{ BMI mean (STD) } \\
\hline Male & $23.6(2.7)$ & $24.4(3.7)$ & $25.0(3.1)$ \\
\hline Female & $22.9(2.4)$ & $24.2(3.4)$ & $23.3(2.3)$ \\
\hline \multicolumn{4}{|l|}{ Race if known (N) } \\
\hline \multicolumn{4}{|l|}{ Male } \\
\hline White & 20 & 12 & 9 \\
\hline Asian & 2 & 10 & 10 \\
\hline African American & 0 & 1 & 2 \\
\hline Hispanic & 4 & 3 & 3 \\
\hline Native Hawaiian & 0 & 0 & 0 \\
\hline \multicolumn{4}{|l|}{ Female } \\
\hline White & 8 & 9 & 5 \\
\hline Asian & 5 & 0 & 1 \\
\hline African American & 1 & 1 & 1 \\
\hline Hispanic & 0 & 0 & 1 \\
\hline Native Hawaiian & 2 & 0 & 0 \\
\hline
\end{tabular}

Since changes in individual lipids can impact health and disease states without necessarily changing an entire lipid class, we compared lipid species across groups using ANOVA with group corrections. Male participants showed a significant change in a number of specific lipids including individual $\mathrm{CE}, \mathrm{PC}$, and HCER mostly driven by an increase in TCIG group when compared with ECIG and non-smokers (Fig. 6a). These results suggest that male ECIG users have a lipid profile that is largely indistinguishable from non-smokers. Similar to male counterparts, female participants showed significant changes in free fatty acids driven by TCIG smokers with no major changes between ECIG or non-smokers for FFA (Fig. 6b). Intriguingly however, a number of plasmalogen species PE (P-16:0/18:2) and PE (P-18:1/18:2) were significantly lower in female ECIG users compared with non-smokers and TCIG smokers (ANOVA followed by multiple comparisons of means at family-wise error rate 


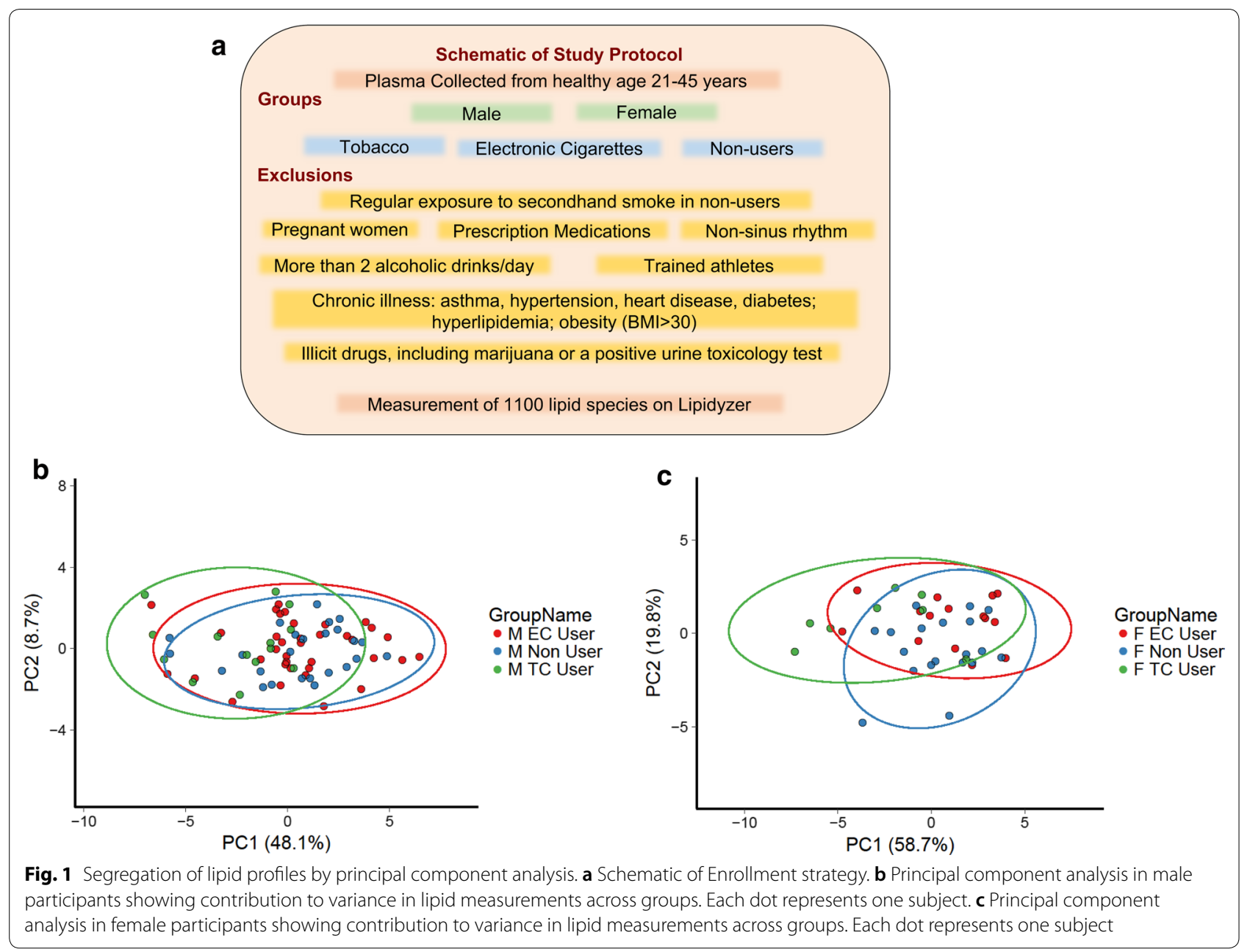

0.05) (Fig. 6b). Comparison of female ECIG and nonsmokers showed significant mean differences in total plasmalogens (ANOVA, $\mathrm{p}=0.02649$ ); with non-smoker mean $73.1 \mathrm{nmol} / \mathrm{ml}$ (95\% CI, 59.98 to 86.31 ) and ECIG mean $54.26 \mathrm{nmol} / \mathrm{ml}$ (95\% CI, 44.61 to 63.90) (Fig. 7). This, results indicate that ECIG use is associated with alterations in lipid species known to be physiologically relevant and that changes associated with ECIG use are independent of and do not necessarily recapitulate changes associated with TCIG smoking.

\section{Discussion}

Our study is one of the first to interrogate changes in lipid species associated with tobacco products in healthy humans. We observed increases in cholesterol esters and fatty acid species with TCIG smoking which is consistent with previous studies [19]. ECIG use, however, does not appear to be associated with dramatic changes in lipid composition although significant alterations in a number lipid species were observed and appear to be independent of nicotine use. Although males and females are known to have differences in lipid species composition, the clinical significance of the sex-specific differences observed in our study are unclear. Intriguingly, a murine study found 

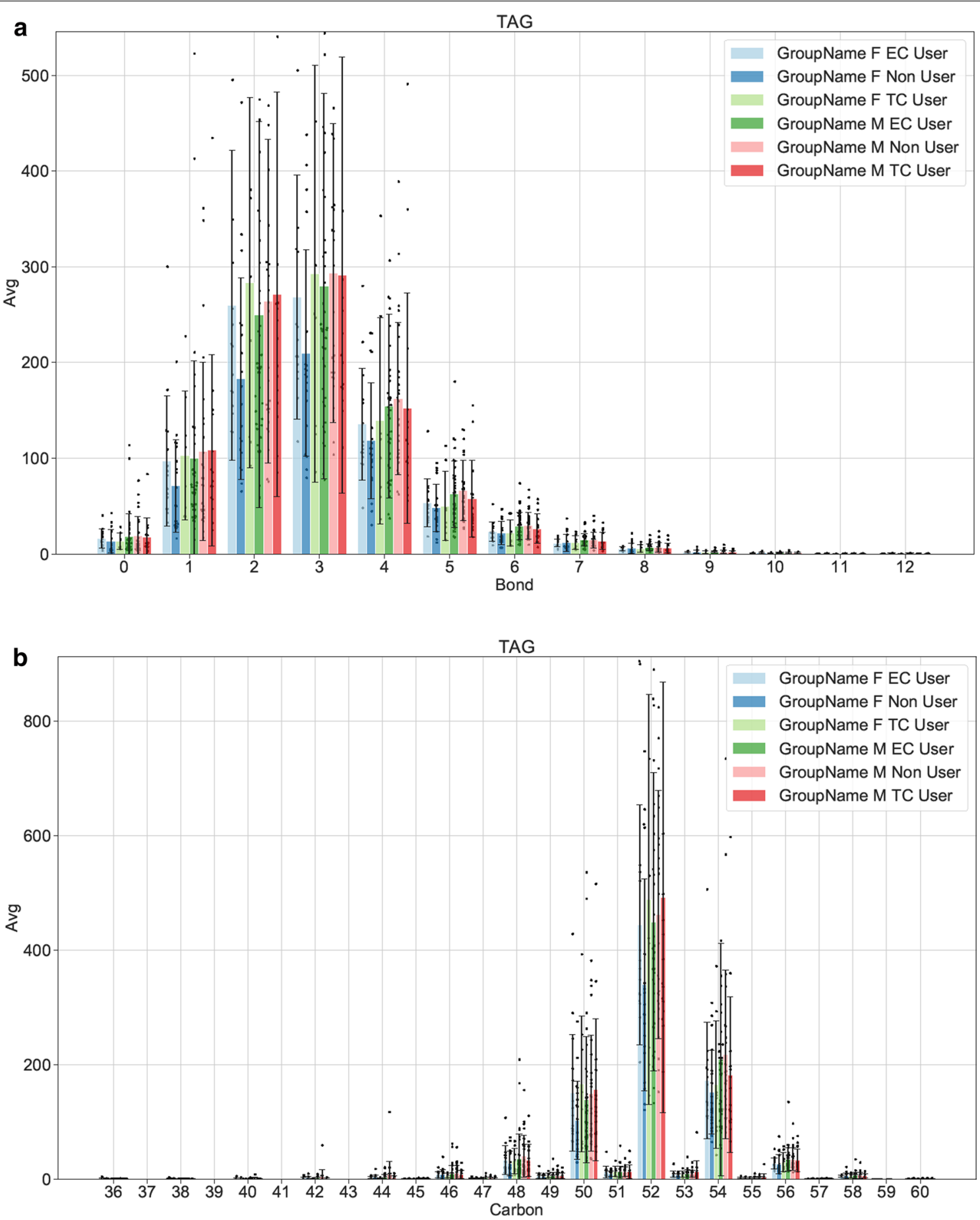

Fig. 2 Levels of Triglyceride across groups. Number of a Bonds and $\mathbf{b}$ Carbons for TAG. Each dot is individual samples measured from plasma. Bar graph is mean \pm SEM 


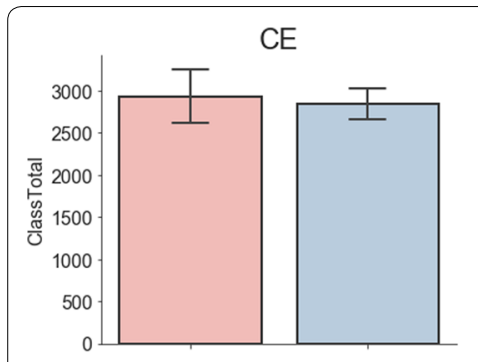

FFA
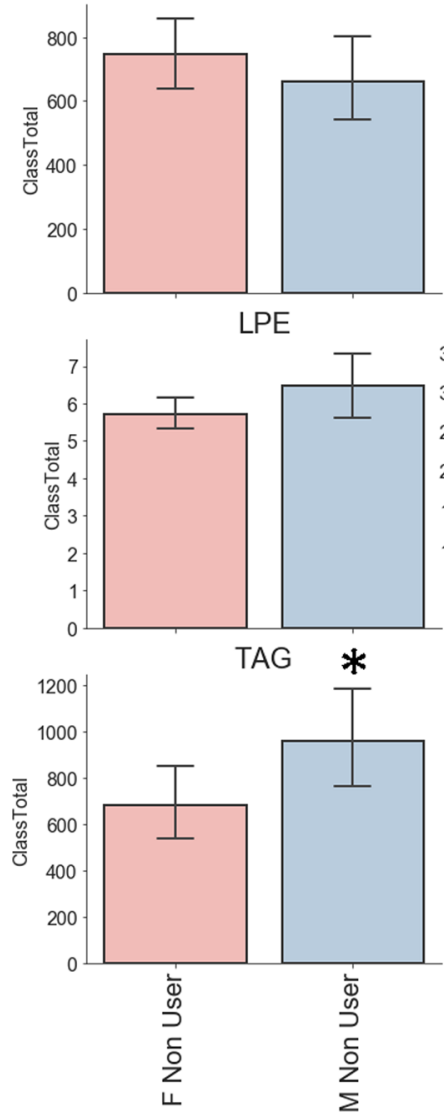

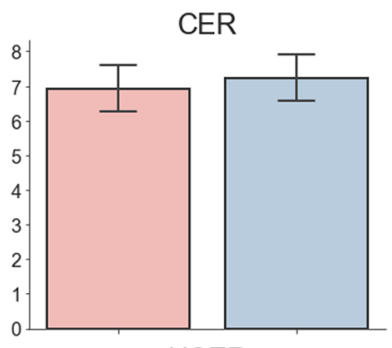

HCER
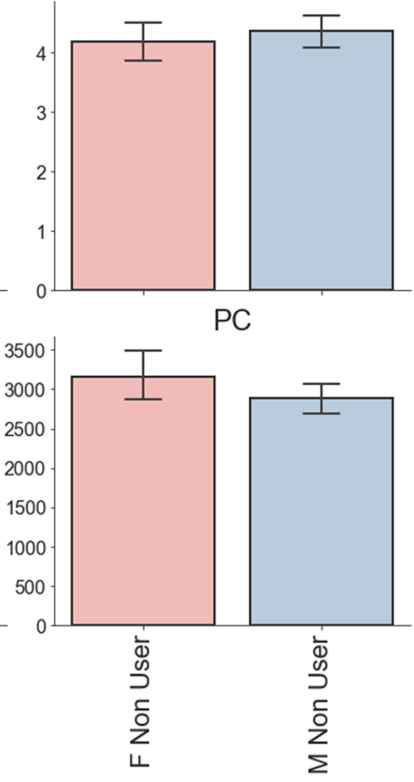

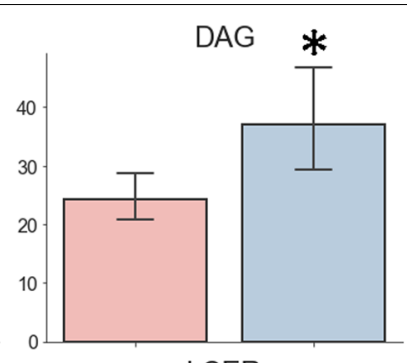

LCER

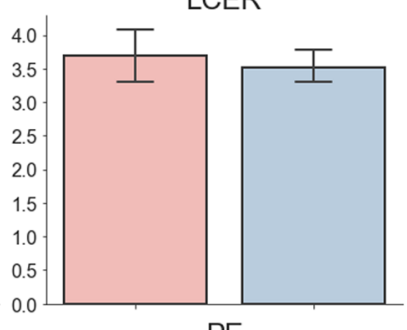

PE
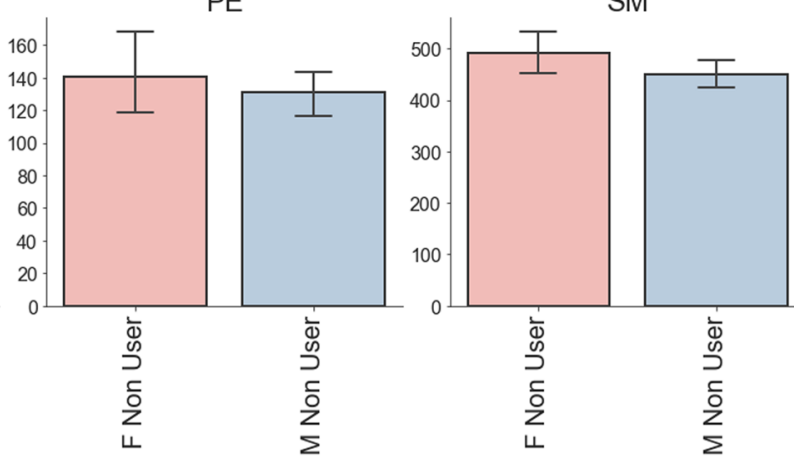

Fig. 3 Major lipid classes from comparing male and female non-users. Each bar graph represents mean $\pm S T D$. *P $<0.01$. CE cholesterol ester, CER Ceramides, DAG diacylglyceroles, DCER dihydroceramides, FFA free fatty acids, HCER hexosylceramides, LCER lactosylceramides, LPC lysophosphatidylcholine, LPE lysophosphatidylethanolamine, PC phosphatidylcholine, PE phosphatidylethanolamine, SM Sphingomyelin, and TAG triacylglycerols

changes in lipid composition associated with ECIG use specifically using female mice [9], however serious complications associated with ECIG use have been reported in predominantly male population although the overall numbers have been small. Although this is one of the largest ECIG studies to date, our sample size is relatively small and tests in larger cohorts of subjects are needed to confirm our findings. In addition, we measured lipid changes in plasma but it is conceivable that ECIG use is associated with local (alveoli) changes in lipids that are not transmitted in plasma or contributions from other organs may be at play. Although most of our study participants reported no medications and oral contraceptive use was reported as minimal (5 subjects) 


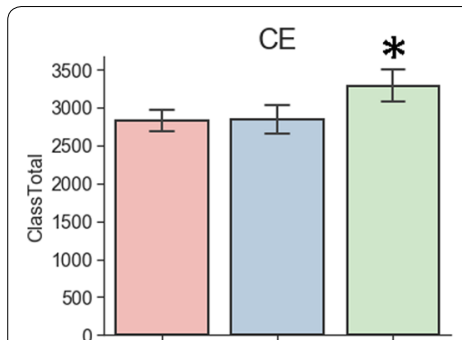

FFA
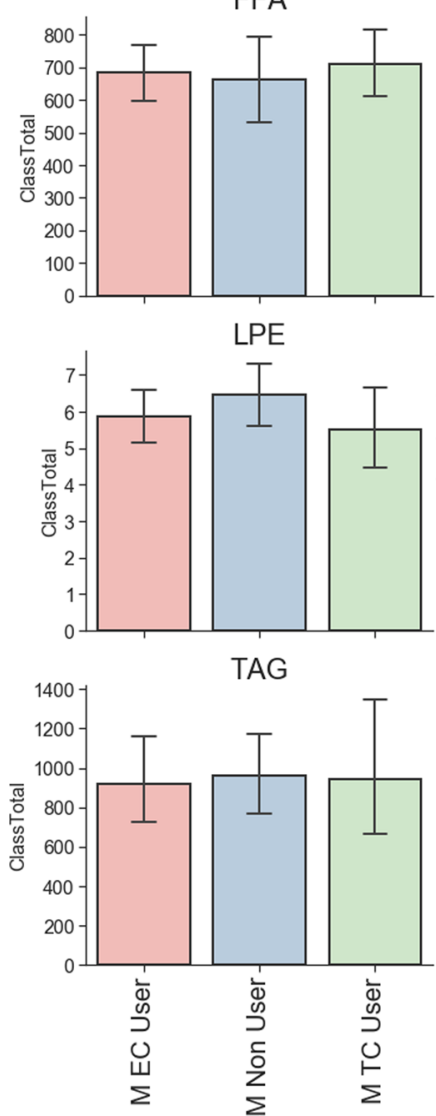

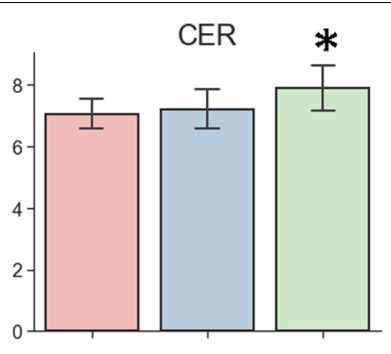

HCER

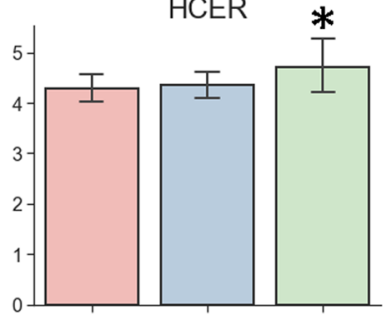

PC
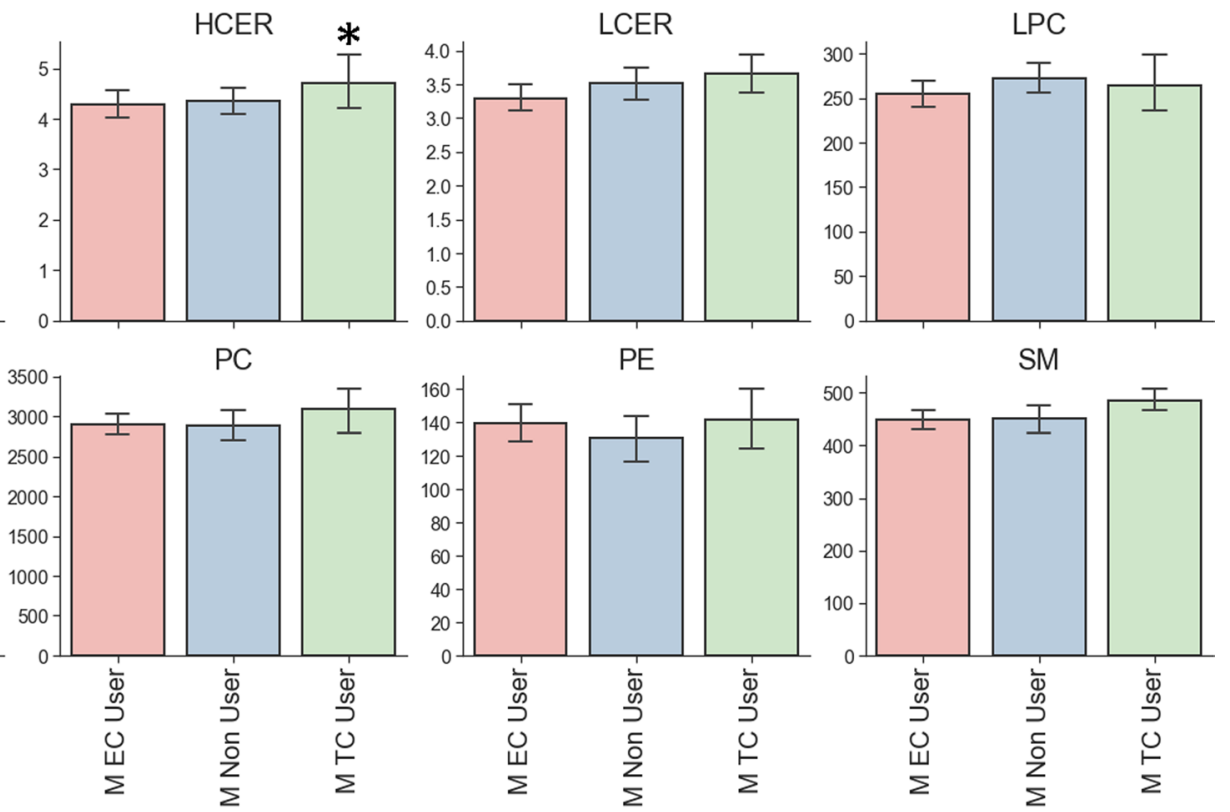

Fig. 4 Major lipid classes from serum samples in male participants. Each bar graph represents mean $\pm S T D$. ${ }^{*} P<0.01$. CE cholesterol ester, CER ceramides, DAG diacylglyceroles, DCER dihydroceramides, FFA free fatty acids, HCER hexosylceramides, LCER lactosylceramides, LPC lysophosphatidylcholine, LPE lysophosphatidylethanolamine, PC phosphatidylcholine, PE phosphatidylethanolamine, SM sphingomyelin, TAG triacylglycerols

it is possible that other medications or supplements may be underreported and could interfere with lipidomic measurements.

Female ECIG users had significantly lower plasmalogen levels compared with non-smokers. Plasmalogens are a class of lipid species that are critical for peroxisome function [13]. Inherited plasmalogen deficiency is associated with Zellweger spectrum and Rhizomelic chondrodysplasia punctate, a syndrome characterized by bone shortening, neurodevelopmental abnormalities, and, importantly, recurrent respiratory tract infections [13]. In addition, a number of studies have reported 

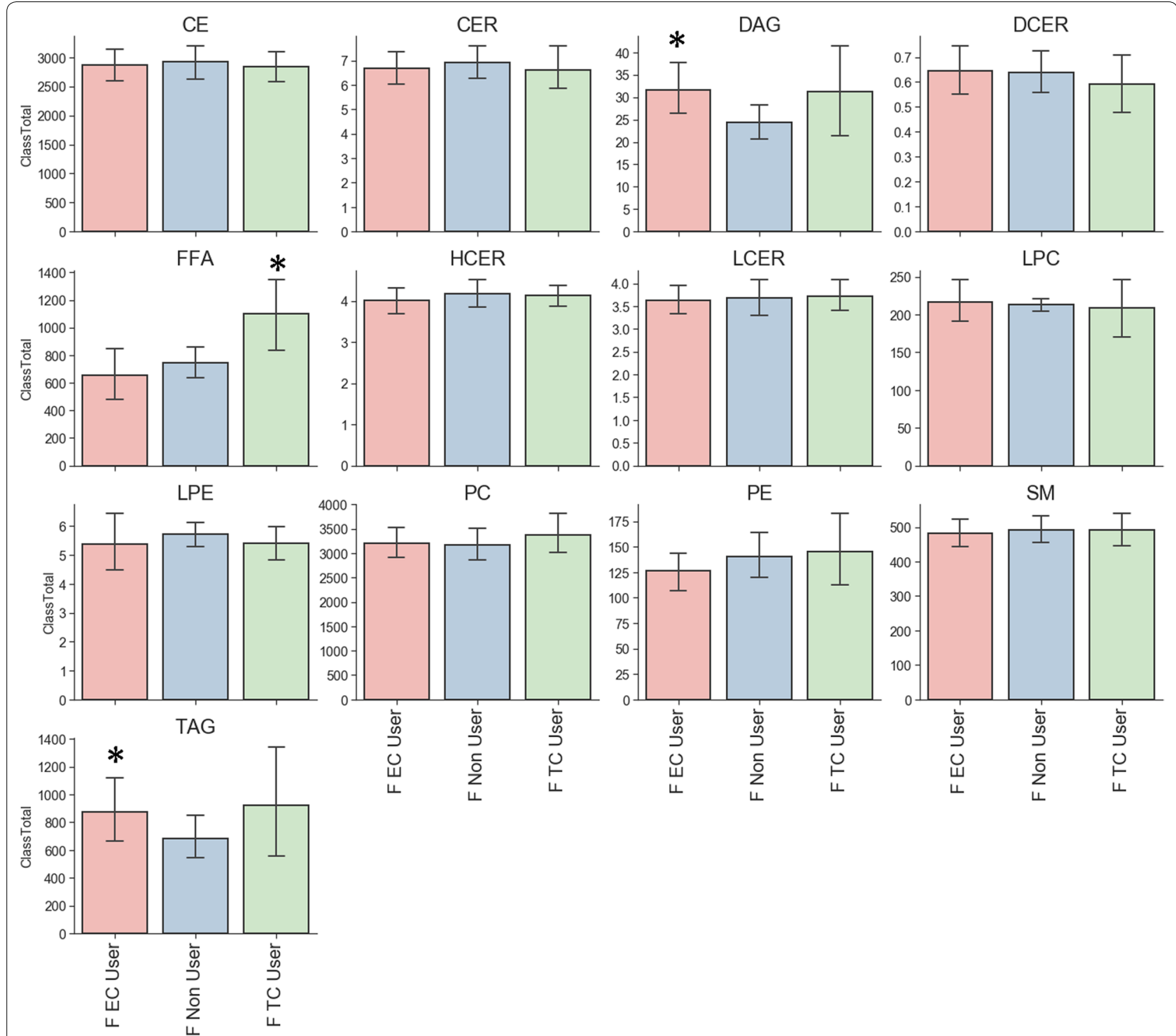

Fig. 5 Major lipid classes from serum samples in female participants. Each bar graph represents mean $\pm S T D$. * $P<0.01$. CE cholesterol ester, CER ceramides, DAG diacylglyceroles, DCER dihydroceramides, FFA free fatty acids, HCER hexosylceramides, LCER lactosylceramides, $L P C$ lysophosphatidylcholine, LPE lysophosphatidylethanolamine, PC phosphatidylcholine, PE phosphatidylethanolamine, SM sphingomyelin, and TAG triacylglycerols 

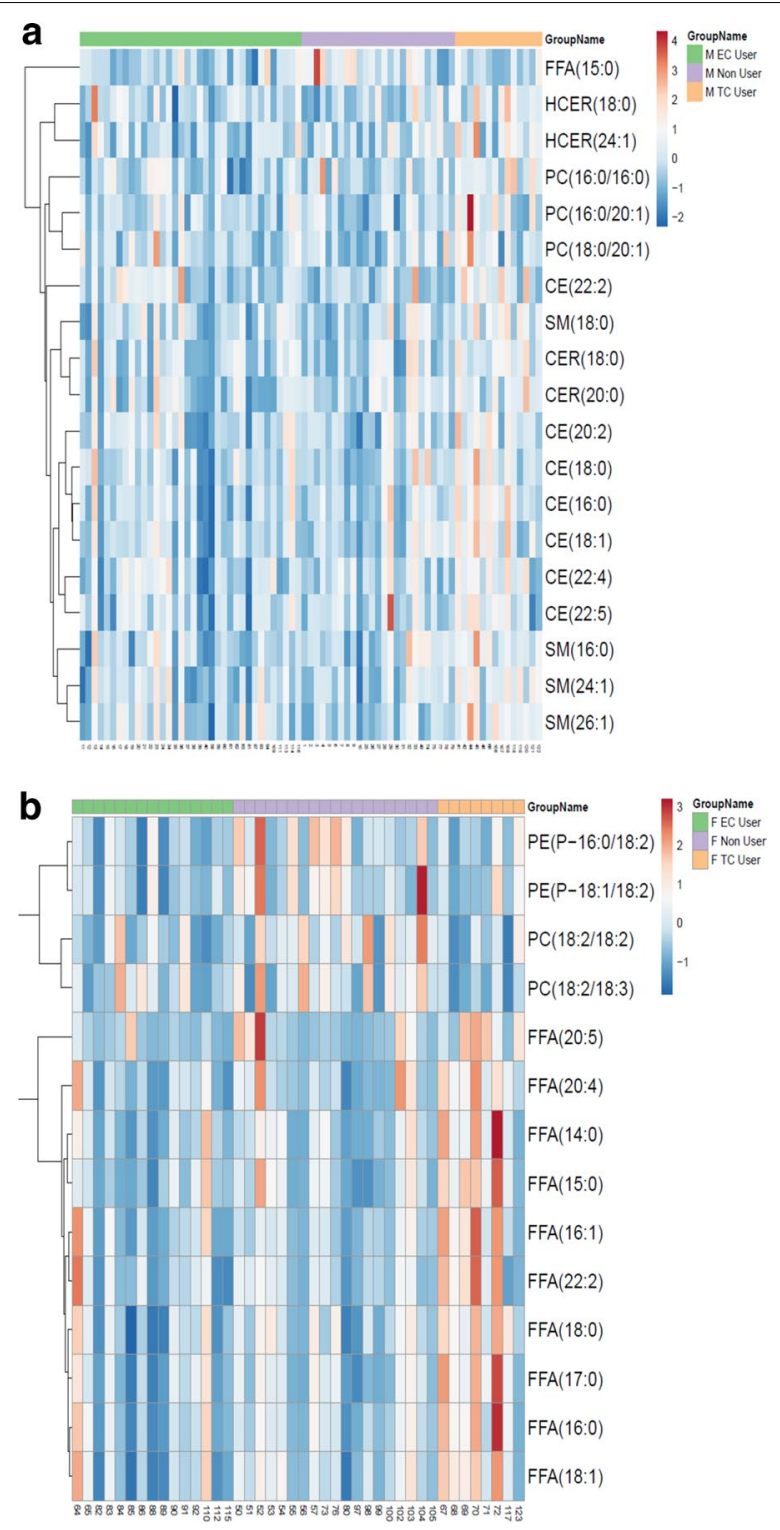

Fig. 6 Individual lipid species differentially measured across groups. Heat map for individual lipids measured across groups for A. Male and B. Female participants. Each species here is statistically significant at $P<0.05$. CE cholesterol ester, CER ceramides, FFA free fatty acids, HCER hexosylceramides, $P C$ phosphatidylcholine, PE phosphatidylethanolamine, and SM sphingomyelin

associations between low plasmalogen levels and respiratory diseases [20]. Plasmalogens are thought to contribute to the proper function of surfactant. Additionally, the lungs are uniquely exposed to ROS and plasmalogens are known to be protective against oxidative stress. Whether the alternations we observed meaningfully contribute to ECIG associated lung injury or other comorbid conditions remains unknown. Future studies will explore this question in greater detail.

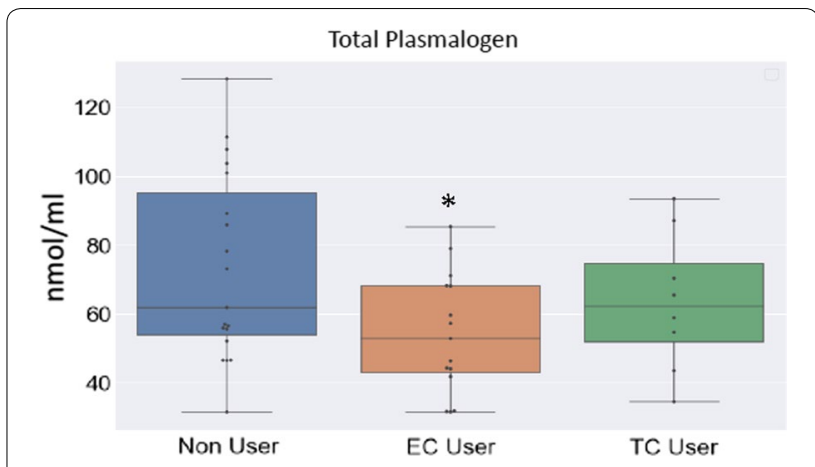

Fig. 7 Evaluation of Plasmalogen levels with electronic cigarette use. The box plots show the distribution of plasmalogen levels in female tobacco non users (Non User), electronic cigarette users (EC) and tobacco cigarette (TC) smokers. The horizontal line in each box represents the median, the lower and upper boundaries of the boxes the interquartile range, and the ends of the bars 1.5 times the interquartile range. ${ }^{*} \mathrm{P}<0.05$

\section{Conclusion}

Out work finds that ECIG use is not associated with global changes in lipid composition but surprisingly specific alterations in serum lipids known to influence normal lung function were observed and this pattern was independent of tobacco use.

\section{Abbreviations}

ECIGS: Electronic cigarettes; TCIGS: Tobacco cigarettes.

\section{Acknowledgements}

We thank members of Sallam and Araujo Labs for their assistance. We also thank Rajat Gupta and UCA CTSI for assistance with sample, collection, processing and storage.

\section{Authors' contributions}

TS and HM conceived the study. TS supervised the study and wrote the manuscript. TS and HM edited the manuscript. HM, KH, JA obtained IRB approval and secured patient enrollment and sample collection. KW and BS measured the different lipids and performed statistical analysis. XW and JK assisted with sample processing. All authors read and approved the final manuscript.

\section{Funding}

This work was supported by NIH grants HL128822 (TS), HL139549(TS), DK118086(TS) and Burroughs Wellcome Fund 469LRRLBWF(TS). Work also supported by the Tobacco-Related Disease Research Program (TRDRP) under the contract numbers: TRDRP 23XT-0006H (HRM), 25IR-0024H (HRM), and TRDRP 28IR-0065 (HRM), and by the NIH National Center for Advancing Translational Science (NCATS) UCLA CTSI Grant Number UL1TR001881.

\section{Availability of data and materials}

Not applicable.

\section{Ethics approval and consent to participate}

We confirm that our study was approved by the Institutional Review Board at the University of California, Los Angeles, and written informed consent was obtained from each participant.

\section{Consent for publication}

The authors consent for publication of this work. 


\section{Competing interests}

The authors declare that they have no competing interests.

\section{Author details}

${ }^{1}$ Division of Cardiology, Department of Medicine, University of California, Los Angeles, CA, USA. ${ }^{2}$ Molecular, Cellular and Integrative Physiology Program, University of California, Los Angeles, CA, USA. ${ }^{3}$ Department of Biological Chemistry, University of California, Los Angeles, CA, USA. ${ }^{4}$ Molecular Biology Institute, University of California, Los Angeles, CA, USA.

Received: 8 July 2020 Accepted: 27 September 2020

Published online: 07 October 2020

\section{References}

1. Pisinger C, Dossing M. A systematic review of health effects of electronic cigarettes. Prev Med. 2014;69:248-60.

2. Levy DT, Yuan Z, Li Y, Mays D, Sanchez-Romero LM. An Examination of the variation in estimates of E-cigarette prevalence among U.S. adults. Int J Environ Res Public Health. 2019;16:3164.

3. Hajek P, Phillips-Waller A, Przulj D, Pesola F, Myers Smith K, Bisal N, Li J, Parrott S, Sasieni P, Dawkins L, Ross L, Goniewicz M, Wu Q, McRobbie HJ. A randomized trial of E-cigarettes versus nicotine-replacement therapy. $\mathrm{N}$ Engl J Med. 2019;380:629-37.

4. Middlekauff HR. Cardiovascular impact of electronic-cigarette use. Trends Cardiovasc Med. 2020;30:133-40.

5. Layden JE, Ghinai I, Pray I, Kimball A, Layer M, Tenforde MW, Navon L, Hoots B, Salvatore PP, Elderbrook M, Haupt T, Kanne J, Patel MT, SaathoffHuber L, King BA, Schier JG, Mikosz CA, Meiman J. Pulmonary illness related to E-cigarette use in Illinois and Wisconsin_-final report. N Engl J Med. 2020;382:903-16.

6. Goniewicz ML, Knysak J, Gawron M, Kosmider L, Sobczak A, Kurek J Prokopowicz A, Jablonska-Czapla M, Rosik-Dulewska C, Havel C, Jacob P 3rd, Benowitz N. Levels of selected carcinogens and toxicants in vapour from electronic cigarettes. Tob Control. 2014;23:133-9.

7. Christiani DC. Vaping-induced acute lung injury. N Engl J Med. 2020;382:960-2

8. Blount BC, Karwowski MP, Shields PG, Morel-Espinosa M, Valentin-Blasini L, Gardner M, Braselton M, Brosius CR, Caron KT, Chambers D, Corstvet J, Cowan E, De Jesus VR, Espinosa P, Fernandez C, Holder C, Kuklenyik Z, Kusovschi JD, Newman C, Reis GB, Rees J, Reese C, Silva L, Seyler T, Song MA, Sosnoff C, Spitzer CR, Tevis D, Wang L, Watson C, Wewers MD, Xia B, Heitkemper DT, Ghinai I, Layden J, Briss P, King BA, Delaney LJ, Jones CM Baldwin GT, Patel A, Meaney-Delman D, Rose D, Krishnasamy V, Barr JR, Thomas J, Pirkle JL, Lung Injury Response Laboratory Working G. Vitamin E acetate in bronchoalveolar-lavage fluid associated with EVALI. N Engl J Med. 2020:382:697-705.

9. Madison MC, Landers CT, Gu BH, Chang CY, Tung HY, You R, Hong MJ, Baghaei N, Song LZ, Porter P, Putluri N, Salas R, Gilbert BE, Levental I,
Campen MJ, Corry DB, Kheradmand F. Electronic cigarettes disrupt lung lipid homeostasis and innate immunity independent of nicotine. J Clin Invest. 2019;129:4290-304.

10. Maddock SD, Cirulis MM, Callahan SJ, Keenan LM, Pirozzi CS, Raman SM Aberegg SK. Pulmonary lipid-laden macrophages and vaping. N Engl J Med. 2019;381:1488-9.

11. Fessler MB, Summer RS. Surfactant lipids at the host-environment interface. Metabolic sensors, suppressors, and effectors of inflammatory lung disease. Am J Respir Cell Mol Biol. 2016;54:624-35.

12. Higenbottam T. Lung lipids and disease. Respiration. 1989;55(Suppl 1):14-27.

13. Braverman NE, Moser AB. Functions of plasmalogen lipids in health and disease. Biochim Biophys Acta. 2012;1822:1442-52.

14. Moheimani RS, Bhetraratana M, Yin F, Peters KM, Gornbein J, Araujo JA, Middlekauff HR. Increased cardiac sympathetic activity and oxidative stress in habitual electronic cigarette users: implications for cardiovascular risk. JAMA Cardiol. 2017;2:278-84.

15. Metsalu T, Vilo J. ClustVis: a web tool for visualizing clustering of multivariate data using Principal Component Analysis and heatmap. Nucleic Acids Res. 2015:43:W566-W570570.

16. Telenga ED, Hoffmann RF, Ruben TK, Hoonhorst SJ, Willemse BW, van Oosterhout AJ, Heijink IH, van den Berge M, Jorge L, Sandra P, Postma DS, Sandra K, Ten Hacken NH. Untargeted lipidomic analysis in chronic obstructive pulmonary disease. Uncovering sphingolipids. Am J Respir Crit Care Med. 2014;190:155-64.

17. Tippetts TS, Winden DR, Swensen AC, Nelson MB, Thatcher MO, Saito RR, Condie TB, Simmons KJ, Judd AM, Reynolds PR, Bikman BT. Cigarette smoke increases cardiomyocyte ceramide accumulation and inhibits mitochondrial respiration. BMC Cardiovasc Disord. 2014;14:165.

18. Holland WL, Bikman BT, Wang LP, Yuguang G, Sargent KM, Bulchand S, Knotts TA, Shui G, Clegg DJ, Wenk MR, Pagliassotti MJ, Scherer PE, Summers SA. Lipid-induced insulin resistance mediated by the proinflammatory receptor TLR4 requires saturated fatty acid-induced ceramide biosynthesis in mice. J Clin Invest. 2011;121:1858-70.

19. Chelland Campbell S, Moffatt RJ, Stamford BA. Smoking and smoking cessation - the relationship between cardiovascular disease and lipoprotein metabolism: a review. Atherosclerosis. 2008;201:225-35.

20. Rudiger M, von Baehr A, Haupt R, Wauer RR, Rustow B. Preterm infants with high polyunsaturated fatty acid and plasmalogen content in tracheal aspirates develop bronchopulmonary dysplasia less often. Crit Care Med. 2000;28:1572-7.

\section{Publisher's Note}

Springer Nature remains neutral with regard to jurisdictional claims in published maps and institutional affiliations.
Ready to submit your research? Choose BMC and benefit from:

- fast, convenient online submission

- thorough peer review by experienced researchers in your field

- rapid publication on acceptance

- support for research data, including large and complex data types

- gold Open Access which fosters wider collaboration and increased citations

- maximum visibility for your research: over $100 \mathrm{M}$ website views per year

At $\mathrm{BMC}$, research is always in progress.

Learn more biomedcentral.com/submissions 